\title{
ESTUDO COMPARATIVO DA CLASSIFICAÇÃO DO GRAU DE PERDA AUDITIVA EM IDOSOS INSTITUCIONALIZADOS
}

\author{
Comparative study for classifying the hearing loss degree \\ in institutionalized elderly
}

\author{
Cristhiane Emy Kano (1), Lais Heloisa Mezzena ${ }^{(2)}$, Heraldo Lorena Guida ${ }^{(3)}$
}

\section{RESUMO}

Objetivo: comparar os resultados audiológicos de idosos institucionalizados, levando-se em consideração duas classificações distintas. Métodos: foram avaliados 40 idosos de ambos os sexos, com faixa etária superior a 60 anos. Após levantar a história clínica audiológica e de realizar otoscopia, foram obtidos os limares tonais (via aérea e via óssea) dos indivíduos, com o uso de audiômetro Eymasa, dentro de cabina acústica. Resultados: demonstraram que a classificação proposta pela Recomendação 02/1 do Bureau International d'Audio Phonologie, foi mais sensível quanto a caracterização da perda auditiva na população idosa. Conclusão: a classificação utilizada pela Recomentação 02/1 é a que melhor representa o grau de perda auditiva nesta população, uma vez que leva em consideração além das freqüências da fala $(500,1 \mathrm{k}$ e $2 \mathrm{kHz})$, a freqüência de $4 \mathrm{kHz}$ para o cálculo da média.

DESCRITORES: Perda Auditiva; Presbiacusia; Idoso; Teste Auditivos; Audição

\section{INTRODUÇÃO}

A Fonoaudiologia busca atender a população idosa em suas diversas necessidades, além de contribuir para a continuidade e qualidade de vida dos mesmos. Afinal, o envelhecimento chegará para todos de uma forma ou outra, e é um processo irreversível ${ }^{1}$. No início do ano de 2001, o Instituto Brasileiro de Geografia e Estatística (IBGE) divulgou que o brasileiro aumentou sua expectativa de vida em dois anos, e o número de idosos cresceu de modo significativo ${ }^{2}$. Em 2003 a expectativa de vida estimada ao nascer no Brasil, para ambos os sexos, subiu para 71,3 anos. Foi um aumento de 0,8 anos em relação à de 2000 , que foi de 70,5 anos $^{3}$. No ano de 2020 espera-se alcançar um total de 32 milhões de pessoas, com mais de 60 anos de idade ${ }^{4}$.

(1) Aluna do Curso de Graduação em Fonoaudiologia da Faculdade de Filosofia e Ciências da Universidade Estadual Paulista, FFC-UNESP, Marília, SP.

(2) Aluna do Curso de Graduação em Fonoaudiologia da Faculdade de Filosofia e Ciências da Universidade Estadual Paulista, FFC-UNESP, Marília, SP.

(3) Fonoaudiólogo; Professor Assistente Doutor do Departamento de Fonoaudiologia da Faculdade de Filosofia e Ciências da Universidade Estadual Paulista, FFC-UNESP, Marília, SP.

Conflito de interesse: inexistente
Presbiacusia é um termo geral referente à perda auditiva no idoso e, como tal, representa a contribuição de um longo período de vida de danos ao sistema auditivo, sendo assim, caracteriza-se por ser uma doença crônica, de etiologia multifatorial ${ }^{5,6}$. Quanto à magnitude de progressão desta perda auditiva, a partir de um estudo longitudinal de limiar de tons puros, foi possível observar um aumento médio aproximado de $1 \mathrm{~dB}$ por ano, para indivíduos com idade igual ou superior a 60 anos ${ }^{7}$. A maioria das perdas auditivas adquiridas em adultos surgem gradualmente e são capazes de dificultar a recepção da linguagem oral ${ }^{8}$.

Entre 50 e 60 anos, a queda do limiar auditivo ocorre mais tarde nas mulheres do que nos homens, e estes apresentam grau de comprometimento auditivo mais acentuado ${ }^{9}$. Além da limitação auditiva, verifica-se na população idosa, o aparecimento do handicap auditivo, relacionado a aspectos não auditivos e às alterações emocionais e sociais desencadeadas pela perda auditiva ${ }^{10}$.

Em Belém - PA, foi realizado um estudo que caracterizou as queixas auditivas e o tipo de perda auditiva em idosos. As principais queixas observadas foram: perda auditiva $(61,7 \%)$, zumbido $(31,5 \%)$ e tontura $(29,6 \%)$. Com respeito aos tipos de perda 
auditiva, os resultados mais relevantes encontrados foram: perda neurossensorial bilateral $(75,3 \%)$, mista bilateral $(7,4 \%)$ e condutiva bilateral $(6,8 \%)^{11}$.

$\mathrm{Na}$ população idosa, há uma prevalência de perda auditiva neurossensorial, bilateral, de configuração descendente, com maior prejuízo nas frequências altas $(4,6$ e $8 \mathrm{KHz})$ para ambas as oreIhas. Os achados dessa pesquisa revelaram ainda que, $32,2 \%$ dos indivíduos apresentaram audição normal, e houve um predomínio de perda auditiva de grau leve $(28 \%)$ e moderado $(25,6 \%)^{3}$. Outra pesquisa sobre a saúde auditiva em idosos, utilizou a média das frequências de $500 \mathrm{~Hz}, 1,2$ e $4 \mathrm{KHz}$, para classificar o grau da perda auditiva. Neste trabalho foi observada maior incidência de perdas auditivas de grau mínimo (41\%), moderado grau 1 (36\%) e moderada grau 2 (20\%) na população idosa avaliada ${ }^{12}$.

Um importante estudo audiológico realizou um levantamento na literatura de dez critérios para classificação do grau de perda auditiva. Os resultados revelaram que, do total de autores pesquisados, sete priorizaram a faixa de frequências de 500 a $2000 \mathrm{~Hz}$, como base de cálculo para a perda média. Os três restantes, apesar de diferirem entre si, priorizaram uma faixa de frequência mais ampla, indo dois deles, até $8000 \mathrm{~Hz}$ e o outro, até $4000 \mathrm{~Hz}^{13}$.

Ao desconsiderar as frequências mais altas, a classificação prioriza a energia dos sons da fala em detrimento de sua inteligibilidade. Um outro fator é que, na maioria das vezes, as perdas auditivas neurossensoriais acometem primeira e principalmente, as frequências altas em vez das baixas, o que contribui para o fato deste critério não explicar as dificuldades comunicativas relatadas pelos indivíduos ${ }^{14}$. Nem sempre o resultado da audiometria tonal corresponde ao resultado encontrado na avaliação do uso funcional da audição pelo idoso. Idosos com perda auditiva leve podem apresentar alto índice de percepção de desvantagem funcional ${ }^{15}$.

O objetivo do presente estudo foi comparar os resultados audiológicos dos idosos institucionalizados, considerando-se a classificação proposta por Davis e Silverman ${ }^{16}$ e a Recomendação 02/1 do Bureau International d'Audio Phonologie - BIAP ${ }^{17}$.

\section{MÉTODOS}

Para a realização da avaliação audiológica foram selecionados 40 idosos residentes no Lar São Vicente de Paulo da cidade de Marília-SP, sendo 22 homens e 18 mulheres, com faixa etária entre 60 e 95 anos. Os idosos selecionados foram submetidos a:
- História clínica audiológica, com objetivo de levantar dados de identificação e a história auditiva do idoso.

- Otoscopia, a fim de verificar a presença de corpo estranho ou qualquer alteração no meato acústico externo e/ou orelha média dos indivíduos, sendo realizado por médico otorrinolaringologista na própria Instituição.

- Audiometria Tonal Liminar para avaliar os limiares tonais (via aérea e via óssea) dos indivíduos, sendo realizado em cabina acústica ${ }^{14}$, instaladas nas dependências da própria Instituição, com o uso de audiômetro Eymasa.

De acordo com as normas preconizadas para pesquisa utilizando seres humanos, este estudo foi analisado e aprovado pelo Comitê de Ética em Pesquisa da Faculdade de Filosofia e Ciências - UNESP / Marília - SP, conforme protocolo $n^{\circ} 3151 / 06$.

Para testar a significância entre grupos (Davis e Silverman ${ }^{16}$ versus BIAP ${ }^{17}$ ) foi realizada a análise comparativa entre as médias dos limiares auditivos com o uso do Teste - ANOVA: Fator Único.

\section{RESULTADOS}

Os resultados da anamnese audiológica revelaram que as principais queixas apresentadas pelos idosos foram: hipertensão arterial em dez (25\%), tontura em sete $(17,5 \%)$, zumbido em sete $(17,5 \%)$ e otalgia em três $(7,5 \%)$.

A otoscopia identificou a presença de cerume em seis (15\%) idosos, nesses indivíduos, foi realizada lavagem do meato acústico externo antes da avaliação audiológica.

A análise dos resultados da audiometria tonal liminar, revelou que todas as perdas auditivas foram tipo neurossensoriais, exceto em um caso, em que foram observadas perda mista à esquerda e neurossensorial à direita.

As Figuras 1 e 2 representam o perfil descendente da presbiacusia, uma vez que há diminuição no número de indivíduos com limiares entre zero e $25 \mathrm{~dB}$, e consequentemente uma piora nos limiares auditivos nas frequências altas $(2 \mathrm{~K} \mathrm{e} 4 \mathrm{KHz})$.

A análise dos resultados do ponto de vista qualitativo (Tabelas 1 e 2), permitiu observar que quando comparadas as duas classificações, verificou-se uma queda em relação ao grau da perda auditiva quando utilizada a classificação BIAP ${ }^{17}$.

No que diz respeito à análise quantitativa, feita após a comparação dos resultados (Davis e Silverman ${ }^{16}$ versus BIAP ${ }^{17}$ ), foi possível observar uma piora significativa para as médias obtidas por meio da classificação BIAP ${ }^{17}$, bilateralmente (Tabelas 3 e 4). 


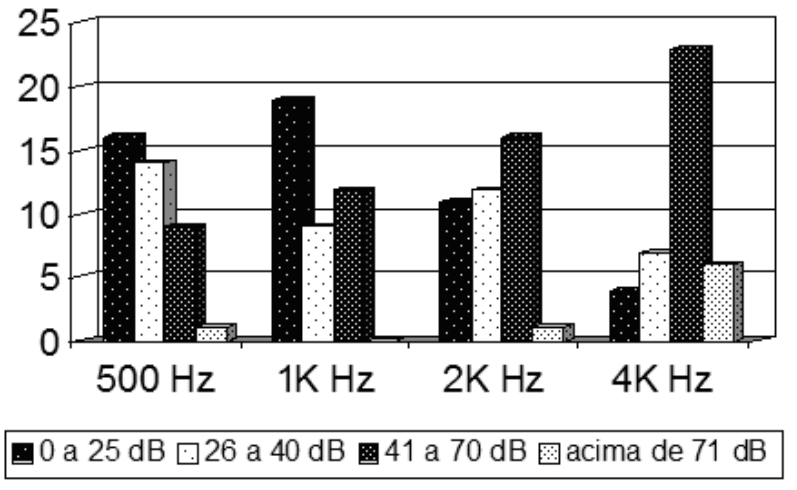

Figura 1 - Distribuição dos resultados da audiometria tonal liminar da orelha direita, nas frequências de 500, 1000, 2000 e $4000 \mathrm{~Hz}$

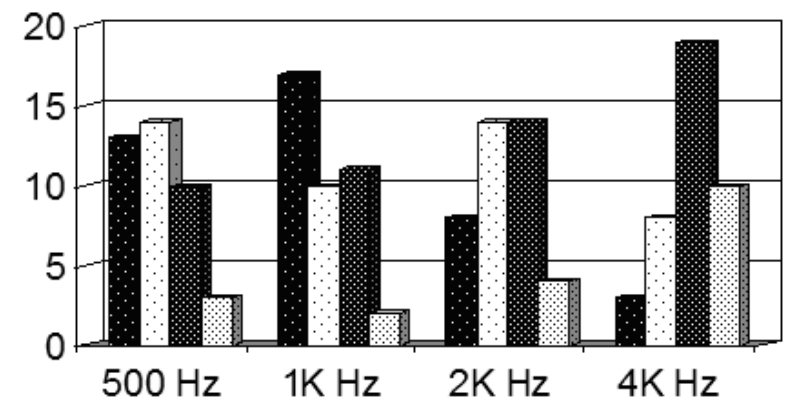

0 a $25 \mathrm{~dB} \square 26$ a $40 \mathrm{~dB}$ 圈 41 a $70 \mathrm{~dB}$ 圈acima de $71 \mathrm{~dB}$

Figura 2 - Distribuição dos resultados da audiometria tonal liminar da orelha esquerda, nas frequências de 500, 1000, 2000 e $4000 \mathrm{~Hz}$

Tabela 1 - Resultados dos achados audiométricos das orelhas direita e esquerda, segundo a classificação do grau da perda auditiva - Davis e Silverman ${ }^{16}$

\begin{tabular}{lcccccc}
\hline \multirow{2}{*}{ Classificação } & \multicolumn{2}{c}{ Orelha Direita } & \multicolumn{2}{c}{ Orelha Esquerda } & \multicolumn{2}{c}{ Total } \\
\cline { 2 - 7 } & $\mathbf{n}$ & $\%$ & $\mathbf{n}$ & $\%$ & $\mathbf{n}$ & $\%$ \\
\hline Normal & 07 & 17,5 & 07 & 17,5 & 14 & 17,5 \\
Leve & 15 & 37,5 & 13 & 32,5 & 28 & 35 \\
Moderada & 16 & 40 & 16 & 40 & 32 & 40 \\
Severa & 02 & 05 & 03 & 7,5 & 05 & 6,25 \\
Profunda & - & - & 01 & 2,5 & 01 & 1,25 \\
\hline
\end{tabular}

Tabela 2 - Resultados dos achados audiométricos das orelhas direita e esquerda, segundo a classificação do grau da perda auditiva - BIAP ${ }^{17}$

\begin{tabular}{lcccccc}
\hline \multirow{2}{*}{ Classificação } & \multicolumn{2}{c}{ Orelha Direita } & \multicolumn{2}{c}{ Orelha Esquerda } & \multicolumn{2}{c}{ Total } \\
\cline { 2 - 7 } & $\mathbf{n}$ & $\%$ & $\mathbf{n}$ & $\%$ & $\mathbf{n}$ & $\%$ \\
\hline Normal & 01 & 2,5 & 01 & 2,5 & 02 & 2,5 \\
Leve & 13 & 32,5 & 11 & 27,5 & 24 & 30 \\
Moderada Grau 1 & 10 & 25 & 07 & 17,5 & 17 & 21,25 \\
Moderada Grau 2 & 10 & 25 & 15 & 37,5 & 25 & 31,25 \\
Severa Grau 1 & 04 & 10 & 02 & 05 & 06 & 7,5 \\
Profunda Grau 1 & 02 & 05 & 01 & 2,5 & 03 & 3,75 \\
Profunda Grau 2 & - & - & 03 & 7,5 & 03 & 3,75 \\
\hline
\end{tabular}

Tabela 3 - Comparação entre os resultados obtidos, segundo as classificações propostas por Davis e Silverman ${ }^{16}$ e BIAP ${ }^{17}$ - orelha direita

\begin{tabular}{lcc}
\hline OD & Davis e Silverman & BIAP \\
\hline Média & 40,26 & $51,82^{*}$ \\
Mediana & 39,58 & 53,99 \\
Desvio Padrão & 15,56 & 18,20 \\
Soma & 1610,33 & 2073,07 \\
\hline
\end{tabular}

* $P=0,003$ significativo para $\alpha=0,05$.
Tabela 4 - Comparação entre os resultados obtidos, segundo as classificações propostas por Davis e Silverman ${ }^{16}$ e BIAP ${ }^{17}$ - orelha esquerda

\begin{tabular}{lcc}
\hline OE & Davis e Silverman & BIAP \\
\hline Média & 45,53 & $56,90^{*}$ \\
Mediana & 40,76 & 58,39 \\
Desvio Padrão & 20,80 & 22,53 \\
Soma & 1821,25 & 2276,22 \\
\hline * $\mathrm{P}=0,021$ significativo para $\alpha=0,05$. &
\end{tabular}




\section{DISCUSSÃO}

Durante a realização do trabalho notou-se uma grande escassez de estudos na área de audiologia relacionada à população institucionalizada, portanto os resultados obtidos poderão ser utilizados por profissionais que atuem com população semeIhante. Segundo um recente estudo ${ }^{18}$, o trabalho audiológico nesta faixa etária da população é muito complexo, e requer do fonoaudiológo a sensibilidade e empatia na compreensão das dificuldades enfrentadas pelo paciente.

No presente estudo, durante anamnese audiológica, os idosos relataram ouvir normalmente, porém durante a realização de todo o procedimento, notouse uma grande dificuldade na compreensão de fala, o que condiz com a literatura ${ }^{19}$, a qual relata que os idosos com déficit de audição, decorrente do processo de envelhecimento apresentam como sintomatologia típica, a dificuldade de compreender a fala, apesar de relatarem ouvir normalmente.

Ainda assim, foi possível identificar os seguintes dados de anamnese audiológica, entre os idosos pesquisados: hipertensão arterial (25\%), tontura $(17,5 \%)$, zumbido (17,5\%) e otalgia $(7,5 \%)$. As queixas de zumbido, tontura e otalgia, são evidenciadas também por outros autores ${ }^{11}$.

Considerando a avaliação audiológica na população idosa, foi observada prevalência de perda auditiva neurossensorial, bilateral, de configuração descendente, com maior prejuízo nas frequências altas para ambas as orelhas ${ }^{3}$, o que condiz com os dados obtidos no presente trabalho e também vai de encontro aos estudos outros pesquisadores ${ }^{20}$.

A análise dos resultados do ponto de vista quantitativo, permitiu observar que quando comparadas as duas classificações, verificou-se uma piora em relação ao grau da perda auditiva, quando utilizada a classificação BIAP ${ }^{17}$. Fato este explicado pelo perfil descendente da perda auditiva na presbiacusia.

Ao desconsiderar as frequências mais altas, a classificação dá prioridade à energia dos sons da fala em detrimento de sua inteligibilidade. Sendo assim, a classificação BIAP ${ }^{17}$ reflete de forma mais adequada, a perda auditiva nos idosos, uma vez que considera a frequência de $4 \mathrm{kHz}$ no cálculo da média ${ }^{14}$.

No presente estudo houve um predomínio de perda auditiva de grau leve (35\%) e moderada (40\%), utilizando-se a classificação proposta por Davis e Silverman ${ }^{16}$. Este dado foi semelhante aos achados de outra pesquisa com idosos, que também evidenciaram este predomínio, mas numa incidência menor - perda auditiva de grau leve
$(28 \%)$ e moderada $(25,6 \%)^{3}$. Estes autores, apesar de utilizarem a classificação Davis e Silverman ${ }^{16}$ em seu estudo, ressaltaram a importância da utilização de uma classificação baseada em diversas faixas de frequências, para determinação do grau da perda auditiva de indivíduos idosos ${ }^{3}$.

Ao realizar a classificação do grau da perda auditiva por meio da classificação BIAP ${ }^{17}$, houve uma diminuição na ocorrência de indivíduos normais (de $17,5 \%$ para 2,5\%), em relação a Davis e Silverman ${ }^{16}$. $E$ as maiores frequências no grau da perda auditiva foram: perda mínima (30\%), moderada grau 1 $(21,25 \%)$ e moderada grau $2(31,25 \%)$. A ocorrência nestes mesmos graus foi também observada por outra pesquisa ${ }^{12}$.

A preservação das frequências baixas nos casos de perda auditiva causada pelo processo de enveInecimento, atenta para a utilização de uma classificação baseada em faixas de frequências mais amplas, que possam caracterizar além da diminuição dos limiares de audibilidade, a real dificuldade do idoso em relação a inteligibilidade da fala.

Nessesentido, a utilização daclassificação BIAP ${ }^{17}$ poderá viabilizar a intervenção clínica com encaminhamento para reabilitação audiológica, mesmo em idosos com audição normal, classificados por Davis e Silverman ${ }^{16}$, mas que na realidade apresentam perdas importantes nas frequências altas.

\section{CONCLUSÃO}

A partir da comparação dos resultados audiológicos dos idosos institucionalizados, considerandose a classificação proposta por Davis e Silverman ${ }^{16}$ e a Recomendação 02/1 do Bureau International d'Audio Phonologie - BIAP ${ }^{17}$, o presente estudo demonstrou que o critério de classificação utilizado pela Recomendação 02/1 do Bureau International d'Audio Phonologie ${ }^{17}$, é a que melhor representa o grau de perda auditiva nesta população, pois possibilita que sujeitos classificados como normais, mas que apresentem diminuição da audição nas frequências altas, sejam classificados com perda auditiva de grau mínimo.

\section{AGRADECIMENTOS}

À Fundação de Amaparoà Pesquisa do Estado de São Paulo - FAPESP (processo no 2006 / 06062-4), pelo auxílio financeiro concedido, e aos Professores Sérgio Koodi Kinoshita, Célia Maria Giacheti e Suely Mayumi Motonaga, pelo apoio na realização do trabalho. 


\begin{abstract}
Purpose: to compare the audiological results for institutionalized elderly, taking into consideration two distinct classifications. Methods: we evaluated 40 elderly of both genders, over 60 -year-old, after assessing the clinical history and conduct of audiological otoscopy, we obtained the pure tone (via air and bone) of the individuals, by using the audiometer Eymasa within an acoustic cabin. Results: the classification used by Recommendation 02/1 of Bureau International d'Audio Phonologie, was more sensitive about hearing loss characterization in the elderly population. Conclusion: the classification used by Recommendation 02/1, is the one that better represents hearing loss degree in this population, since it takes in consideration in addition to the speech frequencies $(500,1 \mathrm{k}$ and $2 \mathrm{kHz})$, the frequency of $4 \mathrm{kHz}$ for calculating the mean value.
\end{abstract}

KEYWORDS: Hearing Loss; Presbycusis; Aged; Hearing Tests; Hearing

\section{REFERÊNCIAS}

1. Greco MC, Russo ICP. Achados audiológicos de indivíduos idosos atendidos em uma clínica particular de São Paulo-SP. Acta ORL. 2006; 24(4):245-54.

2. Russo ICP. Intervenção audiológica no idoso. In: Ferreira LP, Lopes DM, Limongi SC. Tratado de fonoaudiologia. São Paulo: Roca; 2004. p.585-96.

3. Baraldi GS, Almeida LC, Borges ACC. Evolução da perda auditiva no decorrer do envelhecimento. Rev Bras Otorrinolaringol. 2007; 73(1):64-70.

4. Veras RP. Em busca de uma assistência adequada à saúde do idoso: revisão da literatura e aplicação de um instrumento de detecção precoce e de previsibilidade de agravos. Cad Saúde Pública. 2003; 19(3):705-15.

5. Gates AG, Mills JH. Presbycusis. Lancet. 2005; 366(24):1111-20.

6. Stenklev NC, Laukli E. Presbyacusis-hearing thresholds and the ISO 7029. Int J Audiolol. 2004; 43(5):295-306.

7. Lee FS, Matthews LJ, Dubno JR, Mills JH. Longitudinal study of pure-tone thresholds in older persons. Ear Hear. 2005; 26(1):1-11.

8. Marchiori LLM, Rego Filho EA, Matsuo T. Hipertensão como fator associado à perda auditiva. Rev Bras Otorrinolaringol. 2006; 72(4):533-40.

9. Soncini F, Costa MJ, Oliveira TMT. Perfil audiológico de indivíduos na faixa etária entre 50 e 60 anos. Fono Atual. 2004; 7(28):21-9.

\section{DOI: $10.1590 / S 1516-18462009005000024$ \\ RECEBIDO EM: 07/05/2008 \\ ACEITO EM: 03/11/2008}

Endereço para correspondência:

Cristhiane Emy Kano

Rua Municipal, 860 casa 03

São Paulo - SP

CEP: 03661-000

E-mail: crisemykano@yahoo.com.br
10. Marques ACO, Kozlowski L, Marques JM. Reabilitação auditiva no idoso. Rev Bras Otorrinolaringol. 2004; 70(6):806-11.

11. Silva BSR, Sousa GB, Russo ICP, Silva JAPR. Caracterização das queixas, tipo de perda auditiva e tratamento de indivíduos idosos atendidos em uma clínica particular de Belém - PA. Arq Int Otorrinolaringol. 2007; 11(4):387-95.

12. Magalhães ATM, Gomez MVSG. Índice de reconhecimento de fala na presbiacusia. Arq Int Otorrinolaringol. 2007; 11(2):169-74.

13. Momensohn-Santos TM, Russo ICP, BahiloNeves CS, Botelho DL, Martins-Dias FA, Rodrigues FL, et al. Estudo comparativo dos critérios utilizados na classificação do grau da perda auditiva. IX Congresso Brasileiro de Fonoaudiologia, 2001; Guarapari, SC.

14. Momensohn-Santos TM, Russo ICP. Prática da audiológica clínica. 5. ed. São Paulo: Cortez; 2005. $375 p$.

15. Veras RP, Mattos LC. Audiologia do envelhecimento: revisão da literatura e perspectivas atuais. Rev Bras Otorrinolaringol. 2007; 73(1):128-34.

16. Davis H, Silverman RS. Hearing and deafness. Nova York: Rinehart \& Wiston; 1970.

17. Bureau Internacional d'Audio Phonologie. Audiometric classification of hearing impairment: recommendation 02/1, 1997. Disponível em: <http://www.biap.biapanglais/rec021.eng.htm>. Acesso em: 08 jun. 2007.

18. Ruschel CV, Carvalho CR, Guarinello AC. A eficiência de um programa de reabilitaçãoaudiológica em idosos com presbiacusia e seus familiares. Rev Soc Bras Fonoaudiol. 2007; 12(2):95-8.

19. Idrizbegovic $E$. The effects of ageing in the peripheral and central auditory system. Karolinska; Proceedings of the 1st Internacional Congress on Geriatric / Gerontologic Audiology; 2004; Stockholm, Sweden.

20. Mazelová J, Popelar J, Syka J. Auditory function in presbycusis: peripheral vs. central changes. Exp Gerontol. 2003; 38(1-2):87-94. 